\title{
Keratinocytes: innate immune cells in atopic dermatitis
}

\author{
Panjit Chieosilapatham ${ }^{1}$, Chanisa Kiatsurayanon ${ }^{2}$, Yoshie Umehara ${ }^{3}$, Juan Valentin \\ Trujillo Paez ${ }^{3}$, Ge Peng ${ }^{3}$, Hainan Yue ${ }^{3}$, Le Thanh Hai Nguyen ${ }^{3}$, and Francois Niyonsaba ${ }^{4}$ \\ ${ }^{1}$ Chiang Mai University Faculty of Medicine \\ ${ }^{2}$ Institute of Dermatology \\ ${ }^{3}$ Juntendo University School of Medicine Graduate School of Medicine \\ ${ }^{4}$ Juntendo University Graduate School of Medicine
}

August 14, 2020

\begin{abstract}
The skin is a unique immune organ that constitutes a complex network of physical, chemical, and microbiological barriers against external insults. Keratinocytes are the most abundant cell type in the epidermis. These cells form the physical skin barrier and represent the first line of the host defense system by sensing pathogens via innate immune receptors, initiating antimicrobial responses and producing various cytokines, chemokines and antimicrobial peptides, which are important events in immunity. A damaged epidermal barrier in atopic dermatitis allows the penetration of potential allergens and pathogens to activate keratinocytes. Among the dysregulation of immune responses in atopic dermatitis, activated keratinocytes play a role in several biological processes that contribute to the pathogenesis of atopic dermatitis. In this review, we summarize the current understanding of the innate immune functions of keratinocytes in the pathogenesis of atopic dermatitis, with a special emphasis on skin-derived antimicrobial peptides and atopic dermatitis-related cytokines and chemokines in keratinocytes. An improved understanding of the innate immunity mediated by keratinocytes can provide helpful insight into the pathophysiological processes of atopic dermatitis and support new therapeutic efforts.
\end{abstract}

\section{Hosted file}

Complete manuscript.pdf available at https://authorea.com/users/350927/articles/475638keratinocytes-innate-immune-cells-in-atopic-dermatitis 